\title{
Implementation of Gabor Filter on ARM9 Processor
}

\author{
Silpa P.A
}

\begin{abstract}
This work implements gabor filter on ARM9 board.Gabor filters are widely used as band pass filters in image processing for texture analysis,feature extraction, edge detection etc. They have frequency and orientation representation similar to human visual system. They have the property of self similarity that is every filter can be generated from mother wavelet.ARM9 processors have v4t architecture. It has strong arithametical capability. Since the ARM9 has features like camera interface,AHB,Enhanced DSP instruction, the filter imple mented on ARM9 can be used for realtime image processing.This implementation uses Opencv functions Index Terms-filter bank; extraction; gabor kernel;
\end{abstract}

\section{Introduction}

Gabor wavelet was proposed by Dennis Gabor in 1946.He defined the "quantum principle " for information:the timefrequency domain for $1 \mathrm{D}$ signals must be quantised in such a way, there should not be any signal or filter that can occupy

less than a certain minimal area in it. The minimal area which represent trade off between time resolution and frequency resolution has a lower limit in this[1]. He showed that Gaussian modulated complex exponentials provides the best representation. The original Gabor functions are generated with fixed Gaussian. Many image processing tasks can be done using Gabor wavelets. In fact Gabor wavelet can be treated as a magnifying lense for seeing the features of image.J.G Dugman showed that the Gabor coefficient mimic the visual cells of humans[6]. They used in even remote sensing of datas.

ARM processors can be used for image procesing with the support of high computational facility,clock frequency,cycle count,pipeline design and extra peripherals. The used processor system on a chip does not integrate image processor.

The pipelining,storage ,display interfaces and other modules increases the number of simultaneous computations. ARM processors support operating system such as Android,Linux, WinCE,Nucleus, Integrity etc.It has low cost development tools. With simple text editor and GCC we can compile the code.In contrast to the traditional image processing equipments which are bulky,consumes more power,costly

ARM line processors offer a good image processing solution[9].They can perform in realtime also.More over ARM shows adaptability to softawre packages which means we can port packages like matlab and opencv to the ARM[10].

\section{Gabor Wavelet}

Generalized the Gabor function to the following 2D form in order to model the receptive fields of the orientation selective simple cells[6]:

$$
\varphi_{i}\left(y_{i}\right)=\frac{l_{j}^{2}}{\sigma^{2}} \frac{\exp ^{l_{j}^{2} y_{0}^{2}}}{\sigma^{2}}\left[x^{l_{m} y}-\exp ^{\frac{-\sigma^{2}}{2}}\right]
$$

Each $\mathrm{i}$ is a plane wave characterized by the vector li enveloped by a Gaussian function, where is the standard deviation of this Gaussian. The center frequency of ith filter is given by the characteristic wave vector

$$
\mathrm{l}_{i}=\left(\begin{array}{c}
l_{i} x \\
l_{i} y
\end{array}\right)=\left[\begin{array}{ll}
l_{v} & \cos \theta \mu \\
l_{v} & \sin \theta \mu
\end{array}\right] \text { (2) }
$$

having a scale and orientation given by $(\mathrm{lv}$,$) . The first term determines the oscillatory part of the$ kernel, and the second term compensates for the DC value. If we subtract the DC response, Gabor filters show no response to the overall level of illumination.

Nuerophysiological study about the receptive field of simple cells shows that spatial structure of the cells having different size is virtually invarient.Daugman proved that ensemble of simple cells is modeled as a family 
of sampled 2D gabor wavelet in log-polar model .By this process we will get a class equivalent to affine coherent states generated by rotation and dilation. This process of decomposition is called wavelet transform of the image[5]

$$
s_{j}(y)=\int j(y) \psi_{j}\left(y-y^{\mid}\right) d y
$$

Where $\mathrm{j}(\mathrm{y})$ is the image intensity value at $\mathrm{y}$.

Each member of gabor wavelet represents receptive field structure of a simple cell in visual cortex.Gabor decomposition mimics directional microscope with some orientation and scaling senarios.

Gabor wavelet transform is introduced and widely used in computer vision area . U.s government conducted a program to find the best algorithm for facerecognition.In that Gabor wavelet based system was the best one[4].

\section{Implementation Of Gabor Wavelet}

In this work Gabor filter is implemented on opencv.We used the formula Gabor=Gaussian*Fourier multiplication of a harmonic function with Gaussian function gives Impulse function. The filter has both real and imaginary component.We can use either the real or imaginary part seperatly or can be used as a complex number.

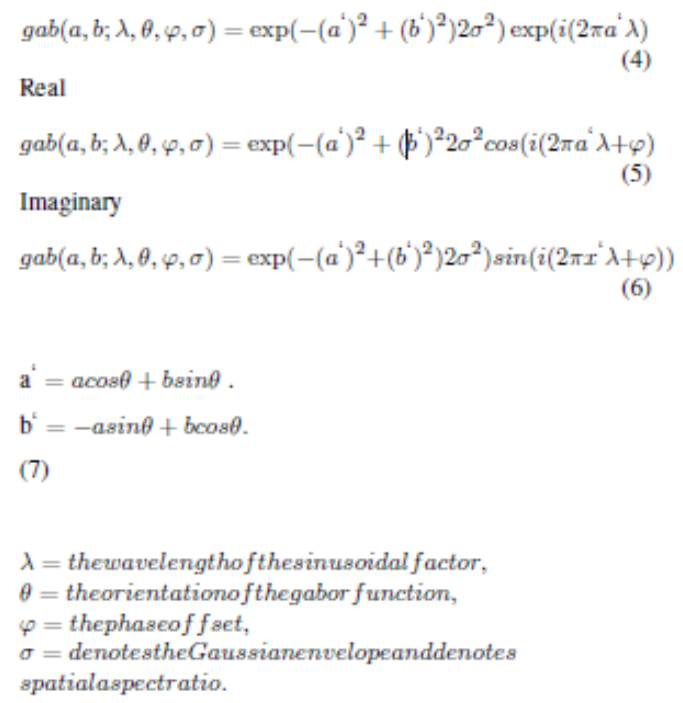

Here the output of the filter may not be an image, it can be a list of values or parameter.Most image processing take the image as two diamensional signal. Here resizing the image is important.Also the image is converted in to the pixel matrix[3]

\section{Result}

Gabor wavelet is implemented on the ARM9 board. The result is as shown in figure.It can be used for edge detection, facerecognition etc.All these are subjects to future work.

TABLE I: Gabor coefficients

\begin{tabular}{cc}
\hline \hline senal number & Coeficients \\
\hline 1 & -00737943 \\
2 & $1.25 \mathrm{E}-04$ \\
3 & $-4.00 \mathrm{E}-06$ \\
4 & $3.46 \mathrm{E}-07$ \\
5 & $5.51 \mathrm{E}-08$ \\
6 & $2.45 \mathrm{e}-12$ \\
7 & $-1.38 \mathrm{e}-11$ \\
8 & $3.55 \mathrm{e}-13$ \\
9 & $7.53 \mathrm{e}-14$
\end{tabular}




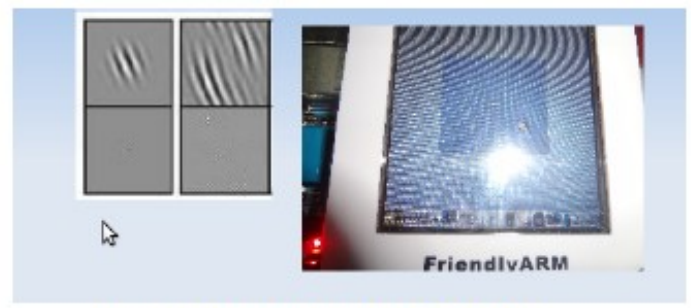

Fig. 1: gabor coefficients generatep on ARM.

\section{References}

[1]. Tenur A,Hosticka .B.J “An adaptive filter for two diamensional gabor transformation," 2010 international conference on image processing and its application, 10.1109/ICASSP.1991.150136

[2]. Kowalczukj,Ebrahim T,Mlynek D ,Kunt M . “A VLSI filter architecture for digital TV codecs," International symposium on circuit and systems 1992,ISCAS’92 10.1109/ISCAS.1992.230293

[3]. Gabor D,Wilby,W.P.L,Wood cock R , . "A universal non-linear filter, predictor and simulator which optimizes itself by learning process" proceedings of IEE-part B;Electronics and communication engineering. vol 108 Issue 40.10.1044/pi-b-2.1961.0070.

[4]. D.M.Tsai, . "Optimal gabor filter design for texture extraction,” D.M Tsai,Yuan -ze university,Chung-Li,Taiwan R.O.C

[5]. Tai sing Lee . "Image representation using 2D gabor wavelet," Pattern analysis and machine intelligence,IEEE transaction on signal processing .10.1109/34.541406.

[6]. wentao Huang ,Zhengping G,Brumpy .P,Kenyon,G.Bettencourt . "development of invarient feature maps via computational model of simple and complex cells," Nueral networks(IJCNN), The 2012 th internationaljoint

[7]. conference on nueral science.

[8]. Christopher Hallinan. "Embedded linux primer," Prentice hall,2006.

[9]. William von Hagen."A definive guide to GCC," 2nd ed,Apress:usa,2006.

[10]. Samsung,Hardware manual for S3C2440(linux).

[11]. Anoop Thomas, Silpa P.A.“Implementation of PCA on ARM9 for face Recognition,"Proceedings of second National conference on emerging trends in Computing ISBN 978-93-82338-64-2, 